\title{
Dynamic Preisach Model Identification Applying FEM and Measured BH Curve
}

\author{
M. Kuczmann \\ Department of Automation \\ Széchenyi István University \\ Győr, Hungary \\ kuczmann@sze.hu
}

\begin{abstract}
Dynamic hysteresis modeling and its application in numerical field analysis are challenging tasks. This paper presents a measurement system to realize and to measure frequency-dependent behavior of ferromagnetic hysteresis. The well-known static Preisach model of hysteresis has been extended to simulate the dynamic behavior. The full paper will present the model in detail as well as its application in the numerical field analysis of the measurement setup.
\end{abstract}

Keywords-magnetic hysteresis; losses, FEM

\section{INTRODUCTION}

From electrical engineering point of view, the aim of measuring hysteresis characteristics of ferromagnetic materials is to perform appropriate input data for numerical implementation and identification of hysteresis models. The realized models can be inserted into electromagnetic field simulation procedures. The electrical devices have various shapes which can be modeled efficiently by Finite Element (FEM) mesh, but the constitutive relation between the magnetic field intensity $H$ and the magnetization $M$ or the magnetic flux density $B$ must be described accurately by hysteresis models. The connection between FEM procedures and hysteresis models can be realized by iterative techniques, one of them is the so-called fixed-point scheme [1], [2], [6].

The rate-independent hysteresis models, such as the original scalar Preisach model, can simulate the $B-H$ relationship at very low frequency. It is known from experiments that the enclosed area of the hysteresis loop, which is the energy loss per cycle, is increasing rapidly by the increase of the frequency of supplied field. The energy loss is a very important technical parameter of soft magnetic materials, and it must be analyzed while developing or designing an electrical device. Rate-independent models in FEM cannot predict losses accurately at higher frequencies, however Maxwell's equations contain the term of eddy currents and their appropriate losses. These losses are smaller than the measured ones. This is the reason why frequency dependency must be taken into account in hysteresis models used in computer aided design applications [3], [4], [5].

The absent losses can be taken into consideration according to the loss separation theory [1], [3]. An extra magnetic field intensity term can be added to the one simulated by the rate-independent model. The loop area, i.e. the loss can be increased in this way. According to experiments, this extra magnetic field intensity is depending on the magnetic flux density $B$ and its variation $\mathrm{d} B / \mathrm{d} t$. There are known techniques to describe this complicated term, [3].

The paper presents a measurement system, containing a toroidal shape core, which can be used to measure static and dynamic scalar hysteresis loops, and a scalar Preisach model to predict these curves as well as the losses. The developed model has been inserted into a two dimensional FEM procedure to analyze the measurement system with axial symmetry. Different kind of motors will also be analyzed.

\section{THE MEASURED CURVES}

The magnetic field intensity inside the analyzed toroidal shape core has been generated by the current $i(t)$ of the primary coil of the core. The arbitrary waveform of current has been built up by applying LabVIEW functions. The time variation of the magnetic field intensity results in a time varying magnetic flux inside the specimen, finally the effect of this flux can be measured by the secondary coil wound on the core. The waveform of magnetic flux density can be prescribed by a simple proportional controller [2].

The measured static hysteresis curves (concentric minor loops) can be seen in Fig. 1, as well as the Everett surface according to an inverse Preisach model. Different dynamic curves can be seen in Fig. 2 measured by the presented system.
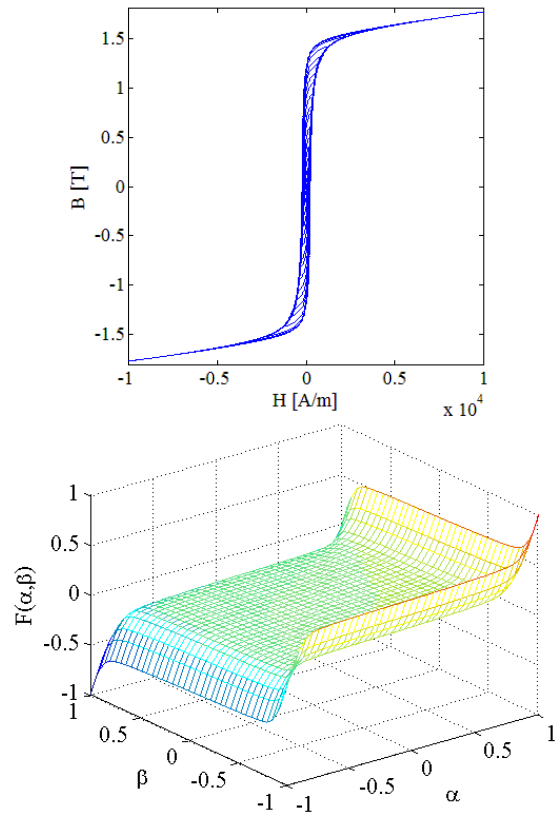

Figure. 1. Static hysteresis loops and the Everett function. 

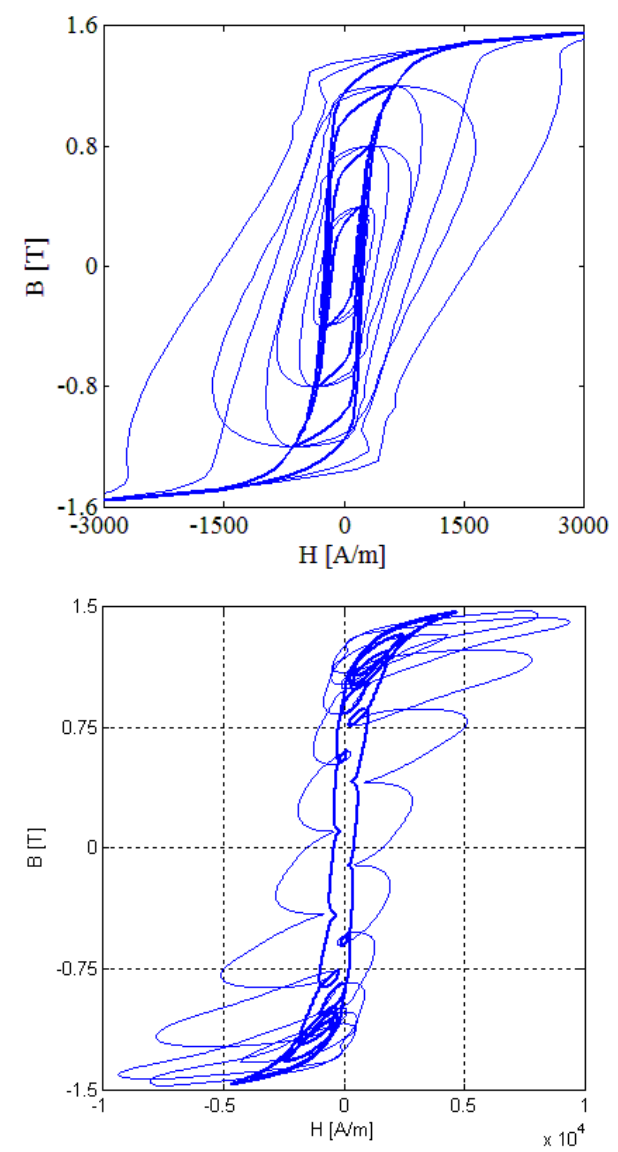

Figure. 2. Measured dynamic hysteresis loops.

\section{DYNAMIC MODEL OF HYSTERESIS}

The investigated frequency-dependent scalar Preisach model is based on the theory that the total loss under sinusoidal magnetization can be decomposed into three terms, i.e. $W_{\text {tot }}=W_{\text {hysteresis }}+W_{\text {classical }}+W_{\text {excess }}$, where $W_{\text {hysteresis }}, W_{\text {classical }}$, and $W_{\text {excess }}$ are the hysteresis loss, the classical eddy current loss, and the excess loss [1], [2], [3]. The hysteresis loss is the area of the hysteresis loop at very low frequency and it is independent of the frequency, the classical eddy current loss is generated by the eddy currents simulated by Maxwell's equations assuming perfectly homogeneous conducting material. This term is proportional to the frequency. According to the experiments, the total loss is usually larger than the sum of these two loss terms. This is why an excess loss term must be added to the sum of hysteresis loss and classical eddy current loss. The last term is proportional to the square of the frequency, and it is depending on microstructural features of the material. If only the frequency-independent Preisach model were used in the simulation, the pure results would be seen on Fig. 3 . The difference between measured and simulated data can be decreased by the use of frequency-dependent Preisach model, as it can be seen on Fig. 3. The full paper will present the developed viscous-type dynamic Preisach model in detail.
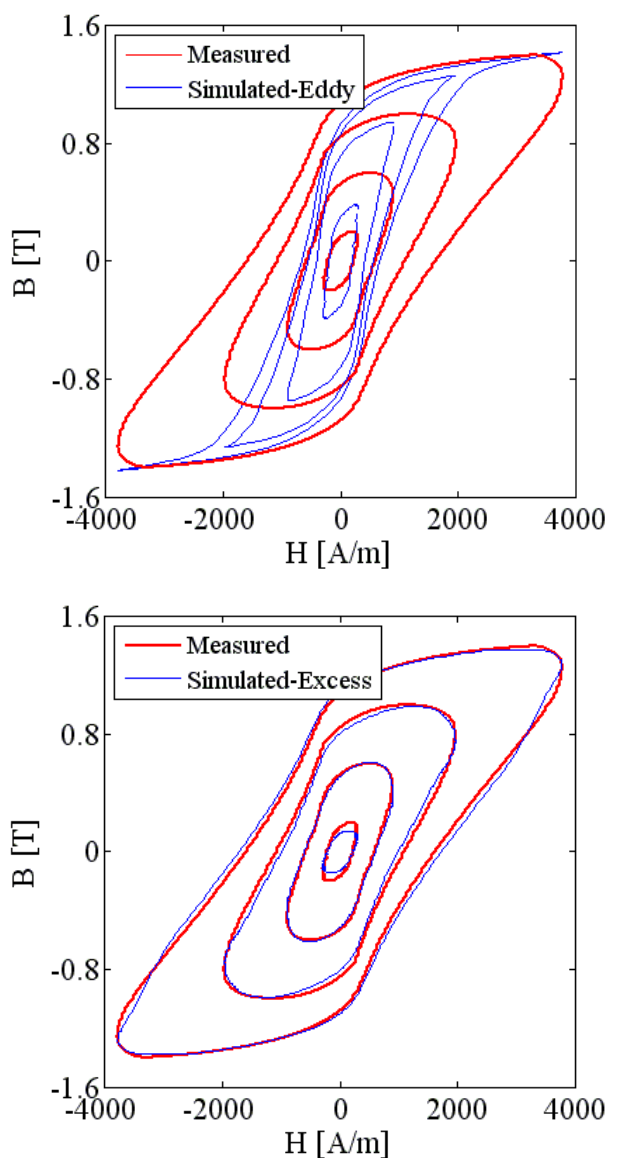

Figure. 3. Comparison of the measured concentric minor loops and the curves simulated by the static model and eddy current field component (up), and the curves simulated by the static model with the eddy current

field component and the excess field component (down).

\section{ACKNOWLEDGMENT}

This paper is sponsored by the Széchenyi István University (15-3202-08), and by "TÁMOP-4.2.2.A11/1/KONV-2012-0012: Basic research for the development of hybrid and electric vehicles - The Project is supported by the Hungarian Government and co-financed by the European Social Fund".

\section{REFERENCES}

[1] G. Bertotti, Hysteresis in Magnetism, Academic Press, 1998.

[2] M. Kuczmann, "Dynamic Preisach hysteresis model,", Journal of Advanced Research in Physics, vol.1, no.1, Paper 011003, 2010.

[3] S. E. Zirka and Y. I. Moroz and P. Marketos and A. J. Moses, "A viscous-type dynamic hysteresis model as a tool for loss separation in conducting ferromagnetic laminations," IEEE Trans. on Magn., vol.41, no.3, pp. 1109-1111, 2005.

[4] P. Marketos and J. P. Hall and S. E. Zirka, "Power Loss Measurement and Prediction of Soft Magnetic Powder Composites Magnetized Under Sinusoidal and Nonsinusoidal Excitation," IEEE Trans. on Magn., vol.44, no.11, pp. 3847-3850, 2008.

[5] E. Dlala and A. Belahcen and A. Arkkio, "On the Importance of Incorporating Iron Losses in the Magnetic Field Solution of Electrical Machines," IEEE Trans. on Magn., vol.46, no.8, pp. 3101-3104, 2010 .

[6] M. Kuczmann and A. Iványi, The Finite Element Method in Magnetics, Budapest: Academic Press, 2008. 